\title{
LA INTERCULTURALIDAD Y LA CONSTRUCCIÓN DE LOS SABERES
}

\author{
Interculturality and the construction of knowledge
}

\author{
Álvaro Alexander Ocampo-González *; Sandra Jenny Otálvaro-Garcés**; \\ Ana Milenia Sánchez.Borrero***
}

Recibido:27 de abril 2020. Aceptado: 27 de junio de 2020. Publicado: 01 de julio 2020.

Forma de citar este artículo en APA:

Ocampo-González, A. A., Otálvaro-Garcés, S. J., Sánchez-Borrero, A. M., (2020, julio-diciembre). La interculturalidad y la construcción de los saberes. Revista CoPaLa, Construyendo Paz Latinoamericana 10 (año 5), pp. 54-68. DOI:10.35600. 25008870.2020.10.0176, Recuperado desde: http://revistacopala.net/index.php/ojs/article/view/24

\section{Resumen}

El presente documento aborda el problema de la interculturalidad y los procesos de aprendizaje en el ámbito de la educación superior. Se consideran algunas formas de aproximación al proceso de aprendizaje, haciendo énfasis en la posibilidad de establecer un punto de partida que oriente el acto educativo. Además, se discute la cuestión que intenta averiguar si solo se aprende a partir de lo que un otro nos enseña en un contexto institucional. De este modo, se resalta la importancia de considerar los presaberes en el proceso de aprendizaje. Por otro lado, se plantea la posibilidad de conocer acerca del estudiante como persona y como perteneciente a un escenario simbólico-cultural para favorecer la construcción de su conocimiento. Finalmente, se propone pensar la interculturalidad a nivel educativo como fuente de flexibilización de las prácticas pedagógicas, como portadora de valores esenciales para crear entornos de aprendizaje y enriquecimiento social.

\section{Palabras clave}

Interculturalidad, Enseñanza, Aprendizaje, Educación.

\footnotetext{
* Psicólogo, Magister en Psicología. Doctor en Ciencias Biomédicas de la Universidad del Valle. Profesor de la Facultad de Salud de la Universidad Santiago de Cali. alvaro.ocampo02@usc.edu.co
** Licenciada en Educación Básica con énfasis en Humanidades y Lengua Castellana. Magister en Tecnología Educativa y Medios Innovadores para la Educación. Profesora, Facultad de Educación a Distancia y Virtual, Institución Universitaria Antonio José Camacho. Profesora de la facultad de educación, Universidad Nacional Abierta y a Distancia (UNAD). sajeotal34@ hotmail.com

*** Licenciada en Literatura de la Universidad del Valle. Magister en Educación: Desarrollo Humano (Universidad San Buenaventura). Profesora, Facultad de educación, Universidad Santiago de Cali. Estudiante de Doctorado en Humanidades, Línea Historia Cultural de Colombia de la Universidad del Valle. ana.sanchez00@usc.edu.co 


\section{Abstract}

This document addresses the problem of interculturality and learning processes in the field of higher education. Some forms of approach to the learning process are considered, emphasizing the possibility of establishing a starting point that guides the educational act. In addition, the question that tries to find out if it is only learned from what another teaches us in an institutional context is discussed. In this way, the importance of considering preknowledge in the teaching-learning process is highlighted. On the other hand, it raises the possibility of knowing about the student as a person and as belonging to a symbolic-cultural scenario to favor the construction of their knowledge. Finally, it is proposed to think interculturality at the educational level as a source of flexibility of pedagogical practices, as a carrier of essential values to create learning environments and social enrichment.

\section{Keywords}

Interculturality, Teaching, Learning, Education. 


\section{Introducción}

La interculturalidad en el contexto educativo se presenta como un componente determinante en el proceso de aprendizaje que construyen los estudiantes en el espacio de la educación superior a partir de una interacción dentro del entorno educativo. Para desarrollar esa idea, como punto de partida se propone la reflexión acerca de la importancia de establecer un acuerdo sobre la concepción que cada escuela erige del proceso de aprendizaje y del sujeto. Posteriormente, se cuestionan aquellas posiciones pedagógicas que tienden a plantear que el aprendizaje solo se logra a partir de lo que un otro nos enseña, específicamente desde las relaciones que se establecen en los ámbitos de enseñanza formales y tradicionales, relegando este proceso a un acto exógeno.

Seguidamente, se considera la función de los presaberes en la estructuración de las relaciones de enseñanzaaprendizaje, reconociendo la importancia práctica del "diagnóstico inicial”" como herramienta fundamental para el docente, facilitando miradas tanto cualitativas como cuantitativas de las posibilidades y el desempeño del educando. Asimismo, se hace énfasis sobre la oportunidad constructiva que reside en el acto de conocer más acerca de la persona del estudiante, incluyendo sus presaberes, su lugar o región de procedencia y las metas que pueda tener, no sólo con respecto a la formación académica sino también en lo relacionado con aspectos de orden social y motivacional. En este sentido, se destaca la práctica de seguimiento de los avances del estudiante, la cual resulta de importancia capital en la medida en que esta permite que el docente se acerque al educando y busque la manera de confirmar, a través de estrategias metodológicas, que está consolidando conocimientos de manera "progresiva". Además, se discute alrededor de la idea según la cual, al pensar el proceso de interculturalidad a nivel educativo, se debe aceptar que el reconocimiento de la diversidad contribuiría a la delimitación y re-configuración de los diferentes contenidos a desarrollar, los núcleos problémicos y las maneras de evaluar los diversos saberes que se ponen en juego en la educación superior.

Finalmente, se plantea que no basta con reconocer la diversidad cultural en los procesos formativos, sino que se hace necesario apreciarla como una fuente significativa de enriquecimiento de las prácticas pedagógicas y de los procesos de todos los educandos en la medida en que es portadora de valores esenciales para crear entornos de aprendizaje y para construir sociedad.

\section{Acerca del aprendizaje}

Desde ciertas concepciones, el aprendizaje ha intentado definirse a través de diversas perspectivas, sin embargo, se pueden considerar algunas aproximaciones que se acercan al fenómeno, dando cuenta de su 
comprensión incompleta y provisional hasta la fecha. Para algunos autores, el aprendizaje consiste en los cambios que se presentan en la conducta como resultado de la práctica que se lleva a cabo en el diario vivir en cualquier aspecto (Beltrán, 1996).

Desde una mirada basada en los principios de la escuela conductista, el aprendizaje sería igual a nivel de cualquier contexto, lo que se entiende por equipotencialidad. De esta manera, las leyes del aprendizaje serían aplicables a diferentes ambientes e individuos, lo que sugiere que sólo existiría una única forma de acceder al aprendizaje y ésta estaría basada en la asociación y en una perspectiva transmisionista (Pozo, 1989).

Esta postura circunscribe las discusiones acerca del aprendizaje a elementos eminentemente predecibles, controlables y medibles, tratando de cumplir con un "requisito de objetividad". En el peor de los casos, el aprendizaje se limitaría a las modificaciones en el comportamiento del aprendiz. Según este supuesto, se asume que el ambiente provee un conjunto de estímulos que aportan a que el educando vaya reforzando una serie de conductas que luego presentará en determinadas condiciones.

Otra definición sobre el aprendizaje hace referencia a los cambios, relativamente permanentes, que el ser humano presenta a nivel de sus capacidades debido a las actividades que realiza, sin que estas sean atribuidas a sus procesos de crecimiento y maduración o relacionadas con patologías. Esto comprende entonces los cambios ocurridos en el individuo, sugiriendo que el aprendizaje nunca deja de producirse (Díaz \& Martins, 1997) y que transforma tanto percepciones como conductas debido a las experiencias vividas (Alonso, Gallego \& Honey, 1997).

De acuerdo con estas miradas, se puede asumir que el aprendizaje es un proceso donde se adquiere conocimientos, habilidades, actitudes y valores mediante la experiencia en diversos entornos, llámese escuela, hogar, trabajo, entre otros, asumiendo una igualdad en la forma de lograrlo, sin delimitar distinciones en los procesos formativos con los que llega cada uno de los sujetos a las situaciones de aprendizaje.

Por otro lado, otras aproximaciones aceptan la idea según la cual el aprendizaje implica modificación de las capacidades inherentes al procesamiento de información de un individuo, lo que nos llevaría a asumir que es afectado por dinámicas subyacentes a los individuos como lo son la percepción, la atención, la memoria, la comprensión entre otros procesos complejos que hacen parte de las denominadas Funciones ejecutivas; esenciales para registrar, procesar, almacenar y resolver situaciones problémicas. 
Otra forma de relacionarse con la idea de aprendizaje es aquella que involucra las posibilidades de hacer de las que puede llegar a disponer el aprendiz, en el sentido de que, los saberes llegan a encontrar utilidad y se inscriben en maneras prácticas que se materializan desde la aplicabilidad e incluso cobran un sentido cultural para el individuo en formación.

Ciertamente, la exploración de aquello que concebimos como proceso de aprendizaje nos ubica en un punto de partida interesante para configurar las prácticas pedagógicas. De acuerdo a Feuerstein (como se citó en De Zubiria, 2006) tanto las metodologías como las didácticas son sutilmente transformables por el modelo pedagógico adoptado, de esta manera se puede afirmar que los modelos heteroestructurantes apelan a las metodologías expositivas y magistrales, al tiempo que los modelos autoestructurantes favorecen actividades como la realización de talleres, excursiones, experimentos, laboratorios, salidas e incluso visitas a museos y desfavorecen en un porcentaje alto la lectura, el diálogo y la exposición docente.

En cuanto a las metodologías interestructurantes estas se relacionan directamente al diálogo y a la valoración de procesos de aprendizaje, lo que implica que estos se llevan a cabo por fases y niveles de complejidad que afirman la mediación del docente frente a la figura del estudiante. De esta manera, se propone llevar a cabo diálogos desequilibrantes que tienen como resultado la desestabilización tomando como referente los presaberes con los que llega el estudiante y valorando la necesidad de la implementación de estrategias pedagógicas como la indagación, el debate, la explicación, entre otros, en la búsqueda de la comprensión, la trascendencia y la mediación del significado.

Sin el ánimo de agotar las posibilidades para abordar la idea de aprendizaje, se hace énfasis en la importancia de asumir que el aprendizaje debe implicar que el individuo llegue a desarrollar una serie de actitudes vinculadas al saber ser, alrededor del conocimiento del que se apropia y que a su vez pueda utilizarlas para su construcción como persona. En otras palabras, se pretende que el aprendizaje parta de una mirada fundamentada desde el sujeto y hacia el sujeto, procurando que él mismo pueda orientar la aplicación de estos saberes en pro del desarrollo del tejido social.

A partir de estas definiciones, se puede inferir que la enseñanza depende del aprendizaje, puesto que, si no hubiese alguien dispuesto a aprender, ¿a quién se le llegaría a enseñar? Para empezar, es posible plantear que ambos conceptos estarían ligados ya que juntos cobran sentido y se integran constituyéndose como el proceso de enseñanza-aprendizaje (Fenstermacher, 1989). 


\section{¿Solo aprendo lo que otro me enseña?}

Al hablar de enseñanza-aprendizaje, Gvirtz \& Palamidessi (1998) afirman que la enseñanza es una "actividad que busca favorecer el aprendizaje. La enseñanza genera un andamiaje para facilitar el aprendizaje de algo que el aprendiz puede hacer si se le brinda una ayuda...” (p. 135). No obstante, aquí se propone una reflexión que pretende cuestionar aquellas posiciones radicales que abierta o implícitamente asumen que solo se aprende a partir de lo que un otro nos enseña, particularmente en el escenario de las relaciones que se establecen en los ámbitos de enseñanza aprendizaje formales y tradicionales. Así, vale la pena lanzar la pregunta de manera concreta: ¿Sólo se aprende a partir de lo que un docente nos enseña de manera formal en un contexto institucional?

En este orden de ideas, se acepta que la participación del otro en tanto probable representante del conocimiento formal que hace parte del acervo cultural e histórico de la humanidad o de los diversos pueblos, se perfila como un elemento importante en el aprendizaje y en el desarrollo que puede llegar a construir un educando en una dimensión particular del conocimiento (Rogoff, 2003; Vigotsky y Kozulin, 1995). Sin embargo, sin desvirtuar el valor social del aprendizaje, se ponen a consideración algunos de los "conocimientos perceptivos" que logramos en la medida en que interactuamos con el mundo. Por ejemplo, en cuanto al aprendizaje visual aprendemos de manera muy temprana a reconocer la profundidad mediante la maduración y las experiencias visuales. Del mismo modo, aprendemos a reconocer cuando un objeto está lejos y cuando está cerca estableciendo relaciones asociadas a su tamaño. Igualmente, llegamos a entender cómo la percepción de velocidad cambia en función de la distancia a la que se encuentra un objeto en movimiento; en este sentido podemos saber que, en términos regulares, un objeto lejano al parecer se mueve más lento, mientras que un objeto cercano nos permite apreciar en él una mayor velocidad en función de nuestros sistemas sensoriales y el modo en que éstos se apropian de la realidad. En cierta medida, podríamos coincidir en que, aunque estos aprendizajes dependen de la experiencia, no están necesariamente ligados a relaciones tradicionales de enseñanza-aprendizaje, ni a los espacios escolares, puesto que el aprendizaje realimente inicia desde el primer día de vida. Si se asume que muchos de los saberes en las diversas dimensiones del conocimiento se estructuran a partir de los aprendizajes perceptivos básicos tanto a nivel visual, táctil, cenestésico como auditivo, entonces podríamos aceptar, aunque sea en una mínima proporción, que los aprendizajes cognitivos más complejos no necesariamente llegan a construirse en un contexto de interacción con un otro de naturaleza formal y escolarizada.

Por tal razón, se acepta que existen datos, piezas de información, saberes y experiencias que el individuo llega a consolidar en una profunda interacción con el ambiente que lo rodea, y desde la dinámica que implica, tanto un proceso biológico madurativo como su participación en un ambiente que le provee estímulos a nivel de lo 
físico, lo sensorial, lo cognitivo y lo social. Este conjunto de hechos, eventos, situaciones y condiciones de vida, se consideran de vital importancia al momento de "emplear métodos de aprendizaje" en las instituciones.

\section{Los presaberes en el proceso de aprendizaje}

Pensar el acto educativo no solamente implica plantearse las relaciones pedagógicas en función de las interacciones que implican el grupo/clase, los objetos del conocimiento y el docente, sino que también involucraría la necesidad de pensar las interacciones desde la particularidad de cada educando, apoyando también una concepción particular de individuo que aprende. Pero en cuanto a la idea del sujeto que aprende, también habría que cuestionarse ésta, siendo que, en una relación de aprendizaje, ambas partes están sujetas a aprender una de la otra, aunque esta situación no se comprende dentro de la concepción de una enseñanza tradicional, en la cual el aprendizaje sería unidireccional.

Lo cierto es que, en estas dinámicas, se debe asumir como elemento fundamental el proceso de diagnóstico inicial que puede llevar a cabo el docente, desde miradas tanto cualitativas como cuantitativas del desempeño del educando. En este orden de ideas, si se acepta que el individuo por sus interacciones con el ambiente y por la asimilación y acomodación de sus propios esquemas mentales puede llegar a modificar aspectos de su inteligencia, también resulta esencial pensar el ser humano como portador de ciertas maneras de ver la realidad y como constructor de ciertas formas explicativas de los fenómenos que lo rodean.

Algunos pensadores razonando a través de una metáfora, han aceptado que el niño pequeño, gracias a la particularidad de su forma de concebir la realidad, se constituye como una especie diferente al humano en su versión adulta. No en vano Rousseau en su libro Emilio, o De la educación presenta un niño que sería evolutivamente diferente al adulto y que debía ser educado teniendo en cuenta sus intereses ${ }^{1}$.

Efectivamente, no se puede asumir al niño como un adulto pequeño tal como se concebía hasta el siglo XVIII. Sin embargo, más allá de la discusión moralizante según la cual "el ser humano nace bueno y es la sociedad quien lo corrompe" (Rousseau,1832), a través de sus investigaciones Piaget (1973) logra evidenciar que las maneras de explicar propias de los niños e incluso las formas particulares de equivocarse que estos presentan, los hacen individuos difíciles de comprender desde la mirada del pensamiento humanamente maduro. En este sentido, la puesta en escena del valor fundamental de los conceptos cotidianos en los procesos de aprendizaje, tanto de la vida diaria como a nivel escolar, resulta esclarecedora en la medida en que estos se lleguen a

${ }^{1}$ Aunque vale la pena aclarar que Rousseau asumía este proceso de una manera muy diferente en lo que respecta al niño y a la niña (a saber, Emilio y Sofía), planteando mayor libertad para el aprendizaje y la proyección a futuro en el hombre que en la mujer. Posición que en la actualidad se perfila como un dato histórico, en términos de una visión justa y respetuosa del valor de los géneros y de su derecho al desarrollo de su potencial en igualdad de condiciones (desde una perspectiva ideal y sin el ánimo de abarcar en esta apreciación todas las singularidades culturales, así como algunas posturas de poder que relativizan el asunto, ya que este problema, complejo por demás, no es parte del propósito de este documento). 
“implicar constructivamente" en la apropiación de los conceptos formales o científicos que aporta la institución denominada escuela a través de las prácticas pedagógicas, que, desde luego se insertan en los imaginarios cultures de los sujetos.

Hoy en día está claro que el individuo no debe asemejarse a un vaso vacío que el profesor llena con los aprendizajes provenientes de su amplio conocimiento y dominio a nivel de determinado campo simbólico. Esto último nos confronta con la idea de un sujeto activo que está más allá de la simple capacidad de respuesta ante los estímulos que provienen del entorno.

Considerar el diagnóstico inicial como una práctica regular al inicio de los diversos cursos de formación en la educación superior, ubica al maestro en una atmósfera de interés por las posibilidades con que llegan los estudiantes y su probabilidad de ser exitosos dentro de los objetivos propuestos para cada asignatura. De esta manera, el diagnóstico inicial le permite al docente realizar ajustes y re-pensar estrategias para garantizar que, habiendo estudiantes con niveles de conocimiento heterogéneos, estos tengan la posibilidad de irse involucrando activamente en la construcción y comprensión de los diversos problemas y conocimientos que se desarrollan en el espacio del aula. Por tal razón, el diagnóstico inicial más que un requisito protocolario se perfilaría como el punto de partida para saber desde qué lugar, a qué velocidad y en qué condiciones "arrancan las diversas naves" que representan cada estudiante desde su experiencia particular y desde su singularidad, considerando que no existe una sola metodología para “enseñar”, ni tampoco una única manera de aprender.

\section{Conocer al estudiante}

Las prácticas pedagógicas deben valerse de diversos modos de abordar el aprendizaje con el fin de que sean inclusivas las maneras particulares que tienen los diferentes educandos para apropiarse del conocimiento. Por tal razón, la acción pedagógica, en tanto didáctica, necesita estructurarse a partir de formatos representacionales variados. En este sentido, ciertas didácticas emplean las habilidades narrativas para abordar la apropiación del conocimiento, o quizás en otras circunstancias el docente decida recurrir a mapas conceptuales, dramatizaciones, juegos, situaciones de resolución de problemas, video foros entre otras didácticas activas que dinamicen el momento educativo, teniendo en cuenta que todo proceso de enseñanza debe partir desde la identificación de quiénes son los que aprenden como brújula para guiar la implementación de estrategias.

Asimismo, se hace énfasis sobre la importancia de conocer más de cerca la persona del estudiante, incluyendo: a) las formaciones previas que este pueda haber realizado, b) su lugar de origen y procedencia y c) las metas que éste pueda haberse establecido, no sólo con respecto a la formación académica sino también en lo concerniente a aspectos de orden social y motivacional. Del mismo modo, se propone realizar un proceso de 
seguimiento a los avances del estudiante, el cual sería favorable para la dimensión formativa, en la medida que el docente se aproxime al educando y busqué la manera de confirmar a través de estrategias metodológicas que, efectivamente el aprendiz sí está consolidando conocimientos de manera exitosa.

Ciertamente, conocer más acerca del estudiante implica contar con la flexibilidad para desligarse de la representación del proceso de enseñanza-aprendizaje como acto unidireccional, y más bien, entenderlo como una relación que se establece cara a cara entre el grupo-clase y el docente. De esta manera, se hace necesario ubicarse en esta misma relación, pero esta vez, considerando al individuo como integrante esencial del vínculo de aprendizaje, qué implicaría al educando (en su individualidad) y al maestro desde sus posibilidades formativas en una relación bidireccional compleja.

Es así como, reconocer aspectos como las formaciones previas que tienen algunos de los estudiantes, por ejemplo, su proceso escolar, profesiones u oficios que han desempeñado en el pasado o quizá incluso en la actualidad, se propone como un aspecto de interés para el docente y para la posterior orientación de su acción pedagógica. Lo anterior, no solamente puede enriquecer la dinámica del curso en términos de los aportes que estos educandos pueden llegar a realizar desde sus vivencias y desde sus saberes, sino también para implicar estas experiencias en las propuestas, vinculándolas a las maneras en que el mismo docente puede llegar a cautivar la motivación y el interés de los diferentes perfiles y formas de sentir que hacen parte de la diversidad en el aula.

El tema de la interculturalidad siempre ha estado presente, como una necesidad urgente en todos los procesos de formación. En este caso, en las instituciones educativas, cada vez más, el asunto de la interculturalidad se constituye como un pilar para quienes lideran una labor educativa, desde el desafío que implica enfrentarse a la heterogeneidad de los colectivos en los cuales, los métodos de enseñanza y evaluación deberían ser acordes a esa diversidad que merece garantizar que nunca perspectivas culturales específicas estén por encima de otras formas de interpretar la realidad propias de otros grupos o individuos. En este sentido, la interculturalidad entendida como espacios de comunicación equitativos entre sujetos de diversas identidades culturales, es una condición propia de aquellos individuos que ingresan a un sistema educativo, en especial, en el siglo XXI. No es un secreto que estas situaciones son cotidianas en el mundo contemporáneo, como escenario de contacto entre sociedades y culturas, y como dinamizador de diálogos, malentendidos, alianzas y conflictos desde los que llegan a constituirse los seres humanos (Grimson, 2001).

Es un hecho que la lógica de la interculturalidad ha crecido de manera significativa, superando las barreras de intolerancia y la simple aceptación del multiculturalismo en la sociedad actual, anexando a las relaciones 
interpersonales elementos esenciales de comunicación que se enmarcan en el diálogo, el entendimiento y la unión entre culturas. Latinoamérica y el Caribe, por ejemplo, cuentan con diversas culturas, con cosmogonías muy particulares, que poco a poco han ido saliendo de sus entornos rurales y han ido perdiendo el temor a enfrentar nuevos retos que las inscriben cada vez más en las rutinas urbanas, por lo que se puede afirmar que, la defensa de la interculturalidad ha permitido a su paso consolidar: a) el conocimiento mutuo entre culturas, b) que exista horizontalidad en las relaciones y c) el respeto por la diferencia. Ciertamente, este proceso no es ajeno a la dinámica de la educación superior, en donde resulta esencial el desarrollo de las relaciones interpersonales de la mano con el reconocimiento de diferencias culturales.

Esta inclusión intercultural hace parte de las instituciones y programas de educación superior, haciéndose presente en las aulas de destacadas universidades que han abastecido sus entornos de saber con miembros de grupos de indígenas y afrodescendientes que durante mucho tiempo han mantenido la lucha por hacer valer su derecho legítimo a la educación. En este sentido, resulta importante reconocer que muchas comunidades cuentan con profesionales que se han preparado específicamente para liderar a su gente y de esta manera, llegar a favorecer el enriquecimiento del nivel de acceso a los saberes. En los espacios universitarios, cada vez es más frecuente el encuentro entre comunidades culturales diversas, que favorecen la apropiación y el relacionamiento con otros desde esas diferencias. En su libro Interculturalidad y comunicación, Grimson describe cuán importante resulta considerar las implicaciones de este fenómeno: "El mundo actual, y quizás cualquier sociedad humana, resulta incomprensible sin atender a las relaciones entre grupos, sociales y culturas. Relación es la palabra clave" (Grimson, 2001, p14).

Además, muchos de estos grupos cuentan con el apoyo de movimientos sociales, ligados al ámbito educativo, que no solo los respaldan, sino que también proponen y llevan a cabo estrategias que permiten que la interculturalidad sea un hecho que favorezca a todos. De este modo, algunos entes gubernamentales, organizaciones no-gubernamentales e incluso fundaciones de carácter privado, asumen determinadas responsabilidades educativas, contribuyendo al proceso de interculturalidad como apuesta social (Aupetit, 2006).

Por tanto, más allá de las formaciones que pueden llegar a tener los educandos y de la heterogeneidad que podrían representar las mismas, el reconocimiento de los orígenes y de la "historia" que trae consigo cada individuo, se constituye como un aporte para la estructuración de prácticas pedagógicas que consideren las diversas realidades y el componente inter-subjetivo en el proceso de aprendizaje. Por tal razón, aproximarse al reconocimiento de las metas y sueños que pueden haberse propuesto los diversos estudiantes, se perfila como una información valiosa que podría favorecer procesos de enganche motivacional que además, pueden llegar 
a involucrar el papel de las emociones en los procesos de apropiación de los diversos saberes. En pocas palabras, se reivindica el valor contenido en el acto de darle espacio, desde los inicios del proceso de enseñanza, al sujeto a través de una mirada que cobije todas sus dimensiones y que cultive esa motivación para hacerlo surgir a partir de los espacios educativos fundados en identidades subjetivas y culturales.

Por otro lado, ayudar a que el estudiante llegue a discriminar entre: a) sensación de aprendizaje, b) aprendizaje y c) comprensión, resulta ser una práctica que le confiere claridad al pedagogo, en pro de la validación de los avances que experimentan los diferentes educandos. Frecuentemente, aún sin saberlo, muchos aprendices pueden llegar a sentir que en un momento dado de la clase aprendieron, pero después de un rato podrían ser "víctimas de esta ilusión", ya que cuando se les confronta nuevamente con el conocimiento es probable que no puedan dar cuenta de lo que supuestamente aprendieron. Así que, la sensación de conocimiento es un mal consejero de la verdadera apropiación de los saberes. Puesto que la real apropiación de los saberes va de la mano con aprendizajes significativos que se logran desde miradas diferentes para orientar la enseñanza (Ausubel, Novak \& Hanesian, 1976).

El aprendizaje en su concepción más tradicional podría llegar a ser reducido a la simple disponibilidad de información memorística con la que cuenta el estudiante y que quizás lo inscriba dentro de maneras de aprendizaje mecanicistas y poco productivas tanto para él, como para su entorno cultural. Por tal razón, se defiende la idea según la cual la comprensión que el individuo logra de los diversos saberes va más allá de la simple disponibilidad de información o formas de proceder frente a las demandas de aplicación formal y lo instaura dentro de la posibilidad de construir nuevo conocimiento, así como plantear posiciones críticas y propositivas frente al conocimiento ya existente. Evidentemente, esto implica una aproximación mucho más flexible frente al proceso de consolidación del aprendizaje.

\section{Acompañar la construcción de los saberes desde la diversidad}

Por último, se plantea como elemento fundamental poder comprender, en su justa medida, la idea según la cual en el proceso de interculturalidad a nivel educativo no basta con reconocer la diversidad, sino que se hace necesario apreciar la misma como fuente de enriquecimiento de las prácticas pedagógicas y de los procesos de todos los educandos, en la medida en que ésta es portadora de valores fundamentales para crear entornos significativos de aprendizaje. Para ello, se acepta que el reconocimiento de la diversidad estaría también delimitando y reconfigurando la deconstrucción y reconstrucción de los diversos asuntos a desarrollar en las asignaturas, los problemas a ser tenidos en cuenta y las maneras de evaluar los múltiples saberes que se ponen en juego en las prácticas correspondientes a la educación superior. Vigotsky (como se citó en Pozo, 1989, p191) plantea esa construcción de saberes y el acompañamiento necesario para ello, considerando "que el 
hombre no se limita a responder a los estímulos, sino que actúa sobre ellos, transformándolos y ello es posible, gracias a la mediación de instrumentos que se interponen entre el estímulo y la respuesta”.

De este modo, asumir con compromiso la idea de interculturalidad como un valor fundamental para la construcción colectiva de conocimientos, resultaría ser uno de los ejes más importantes del proceso de enseñanza-aprendizaje. De lo contrario, la interculturalidad quedaría reducida a una especie de discurso vacío, constituyéndose como un simple adorno del cual se revisten ciertas prácticas pedagógicas para validarse como a) sofisticadas, b) humanizantes y c) ubicadas a la vanguardia de lo que las ciencias de la educación y las ciencias humanas proponen en la actualidad.

Por tal razón, tomarse en serio la interculturalidad implica no sólo reconocerla, sino apreciarla y entremezclarla como un valor esencial de la práctica pedagógica que invita necesariamente al maestro a delimitar de manera diferente los diversos problemas o núcleos conceptuales, alrededor de los que girarán sus acciones formativas, así como ajustar las metodologías para el desarrollo y la construcción conjunta de los saberes teniendo en cuenta las relaciones educando-educando, maestro-educando y de los diversos agentes que participan en la construcción del conocimiento. A su vez, es importante transformar el paradigma según el cual, en los espacios educativos quien "aprende" es solo el estudiante, dejando por fuera del proceso al docente, cuando en la actualidad se reconoce que éste a través de la lectura, del debate y del relacionamiento con sus estudiantes, también va construyendo y deconstruyendo su conocimiento.

Del mismo modo, al considerar seriamente la interculturalidad en las prácticas pedagógicas, el proceso valorativo y evaluativo traería al escenario tanto las metodologías cuantitativas como cualitativas, las cuales se ajustarían en términos de las necesidades culturales que presentan los diversos miembros del grupo-clase y de la obligación humana que apremia al docente frente a su compromiso de lograr que los diversos estudiantes avancen en el proceso de configuración de los saberes. Proceso que además favorecería formas sociales de participación guiada (Rogoff, 1997) que delimitarían caminos para acercarse tanto al saber cómo a la expresión creativa basada en los efectos culturales que emergen de la relación con los otros (Glăveanu, 2010; Nouri, et al., 2015).

La interculturalidad, trasciende los espacios educativos y se instaura en lo social, económico, político, y demás dimensiones, proyectando en éstas las maneras de comportarse frente a ese fenómeno. Grimson precisa que ésta "abarca un conjunto inmenso de fenómenos que incluyen la convivencia en ciudades multiétnicas... Estados multiétnicos, proyectos empresarios, el turismo, la vida fronteriza y los medios masivos de comunicación, entre otros" (Grimson, 2001, p.15). 
Finalmente, considerar la interculturalidad como aspecto importante del proceso educativo, de alguna manera, se reflejaría en el aporte colectivo que implica que los miembros de diversas culturas que hayan pasado por la experiencia de escolarización tengan la oportunidad de participar en procesos de cambio y transformación del tejido social, aportando desde el pensamiento divergente y la acción creativa a la construcción de un mundo mejor. Para lograr esto último es importante entender que al involucrar la interculturalidad en el acto educativo se debe ir más allá de posiciones egocéntricas, que se cubren con la "bandera de la diversidad", para estar a la vanguardia de lo que se trabaja en las ciencias de la educación, para responder a pedidos institucionales o para garantizar espacios que aporten a la perpetuación del ejercicio del poder. Estas agendas ocultas, que se alejan del bien común para instaurarse en el terreno de los intereses personales de un docente con una política equivocada y acomodada a su conveniencia, hacen que las futuras reflexiones deban apuntar a pensarse la interculturalidad y el aprendizaje más allá del envoltorio del poder.

\section{Conclusiones}

Para abordar el problema de la interculturalidad y los procesos de aprendizaje en el ámbito de la educación superior, es necesario revisar múltiples aspectos que hacen justicia a la naturaleza compleja del asunto. De esta manera, las formas de aproximarse al proceso de aprendizaje, contribuyen al establecimiento de un punto de partida que orienta el sentido del acto educativo como acción transformadora. Esta acción transformadora debe iniciar con una mirada que incorpore la diversidad de los sujetos, su identidad cultural y sus formas pensar, apostándole a un aprendizaje basado en este encuentro y no en sistemas tradicionales transmisivos que sean ajenos a estas dinámicas.

Resulta conveniente aclarar que, sin desestimar el profundo valor social del proceso de aprendizaje, no solo se conquistan los saberes a partir de lo que un otro le enseña al individuo en un contexto institucional que le apuesta a determinadas formas de elaboración de los saberes. Se podría decir que un aprendizaje significativo se elabora desde los intereses y motivaciones de los sujetos. Así el docente se convierte en un mediador de la construcción de saberes.

También, se confiere valor al lugar de los presaberes en el proceso de enseñanza-aprendizaje que, de manera práctica se abren a la mirada del docente desde los procedimientos y relaciones establecidas en aquello que se conoce como “diagnóstico pedagógico inicial”. Es así que, la posibilidad que se brinda el maestro para conocer al estudiante desde su persona, sus posibilidades cognitivas y su cosmogonía, favorecen la construcción del conocimiento del individuo en formación y contribuyen a la delimitación y despliegue del acto educativo y la experiencia de aprendizaje, lo que constituye la interculturalidad como matriz esencial de las relaciones pedagógicas en pro del aprendizaje y la construcción del espacio social. 
De esta manera, la interculturalidad tiene que dejar de ser una palabra que se utiliza en todos los discursos de manera formal y conveniente, para el caso, en el campo educativo. Una palabra que en cierta medida ha sido mal utilizada, quedando escindida de la aplicación en hechos cotidianos y conscientes. Los procesos de enseñanza y de evaluación, estarían llamados a propender su avivamiento, generando verdaderos espacios de re-conexión con la diversidad de nuestros pueblos.

\section{Referencias bibliográficas}

Alonso, C. M., Gallego, D. J., \& Honey, P. (1997). Los estilos de aprendizaje: procedimientos de diagnóstico y mejora. Bilbao, España: Mensajero.

Aupetit, S. D. (2006). Pathways to Higher Education: una oportunidad de educación superior para jóvenes indígenas en México. Anuies.

Ausubel, D. P., Novak, J. Y. H. H., \& Hanesian, H. (1976). Significado y aprendizaje significativo. Psicología educativa: un punto de vista cognoscitivo, 53-106. https://cmapspublic2.ihmc.us/rid=1J3D72LMF1TF42P4-PWD/aprendizaje\%20significativo.pdf

Beltrán, J. (1996). Procesos, estrategias y técnicas de aprendizaje. Madrid: Síntesis.

Samper, J. D. S. (2011). Los modelos pedagógicos: hacia una pedagogía dialogante. https://biblioteca.uazuay.edu.ec/buscar/item/80424

Díaz Bordenave, J., \& Martins Pereira, A. (1997). Estrategias de enseñanza-aprendizaje (No. IICA C10-57). IICA (Costa Rica).

Fenstermacher, G. (1989). Tres aspectos de la filosofía de la investigación sobre la enseñanza. $L a$ investigación de la enseñanza: enfoques, teorías y métodos. Barcelona: Paidós.

Glăveanu, V. P. (2010). Paradigms in the study of creativity: Introducing the perspective of cultural psychology. New ideas in psychology, $28(1)$ 79-93. https://www.sciencedirect.com/science/article/abs/pii/S0732118X09000415

Grimson, A. (2001). Interculturalidad y comunicación (Vol. 7). Editorial Norma.

Gvirtz, S., \& Palamidessi, M. (1998). El ABC de la tarea docente: currículum y enseñanza (Vol. 1). Buenos Aires: Aique. http://www.terras.edu.ar/biblioteca/35/35GVIRTZ-Silvina-PALAMIDESSI-MarianoSegunda-parte-Cap-6-La-planificacion.pdf

Nouri, R., Erez, M., Lee, C., Liang, J., Bannister, B. D., \& Chiu, W. (2015). Social context: Key to understanding culture's effects on creativity. Journal of Organizational Behavior, 36(7), 899-918. doi: 10.1002 / job.1923 https://onlinelibrary.wiley.com/doi/epdf/10.1002/job.1923

Piaget, J. (1973). La formación del símbolo en el niño. Fondo de cultura económica.

Pozo, J.I (1989). Teorías cognitivas del aprendizaje. Ediciones Morata. Madrid. http://www.terras.edu.ar/biblioteca/6/6TA_Pozo_2_Unidad_1.pdf 
Rogoff, B. (1997). Los tres planos de la actividad sociocultural: apropiación participativa, participación guiada y aprendizaje. La mente sociocultural. Aproximaciones teóricas y aplicadas, 111-128.

Rogoff, B. (2003). La naturaleza cultural del desarrollo humano. Prensa de la Universidad de Oxford. Rousseau, J. J. (1832). El contrato social, ó Principios del Derecho político.

Vygotsky, L. S., y Kozulin, A. (1995). Pensamiento y lenguaje. (pp. 97-115). Barcelona: Paidós. http://www.academiaperuanadepsicologia.net/libros\%20de\%20epistemologia/Vygotsky-ObrasEscogidas-TOMO-2.pdf 